\title{
Simulation of extreme weather events by a stochastic weather generator
}

\author{
Mikhail A. Semenov* \\ Centre for Mathematical and Computational Biology, Rothamsted Research, Harpenden, Herts AL5 2JQ, UK
}

\begin{abstract}
A stochastic weather generator is a model capable of generating daily weather patterns that are statistically similar to the observed patterns. Weather generators are commonly used in climate change studies as a computationally inexpensive tool to generate high resolution climate change scenarios based on the output from global climate models. Considering that the frequency and the magnitude of extreme weather events are likely to increase under climate change, there is a growing need to investigate how well weather extremes are simulated by weather generators. The aim of this study was to test the skill of the LARS-WG stochastic weather generator to simulate extreme weather events at 20 locations with diverse climates. Yearly maxima of daily precipitation, maximum temperature and length of heat waves, and their 10 and 20 yr return values were compared for observed and synthetic data by fitting the generalized extreme value distribution and computing confidence intervals. Means of yearly maxima and return values of daily synthetic precipitation were within the $95 \%$ confidence intervals $\left(\mathrm{CI}^{95}\right)$ of observed data for all sites. Daily maximum temperature extremes were reproduced less accurately. Although the root mean squared error (RMSE) calculated for the means of maxima of maximum temperature was $<1^{\circ} \mathrm{C}$, synthetic means for approximately half of the sites were outside the $\mathrm{CI}^{95}$ for observed values. This indicates that the assumption used in LARS-WG, that daily temperature could be approximated by the normal distribution, is inadequate. Means of yearly maxima for length of heat waves and 10 and $20 \mathrm{yr}$ return values were within the $\mathrm{CI}^{95}$ for all sites except 3. For those sites where LARS-WG performance was inadequate, daily maximum temperature was not normally distributed but was skewed.
\end{abstract}

KEY WORDS: LARS-WG $\cdot$ Generalized extreme value distribution $\cdot$ Diverse climates $\cdot$ Validation

\section{INTRODUCTION}

A stochastic weather generator (WG) is a model generating daily weather time series statistically similar to the observed weather (Wilks \& Wilby 1999). WGs were adopted in climate change impact studies as a computationally inexpensive tool to generate scenarios with high temporal and spatial resolutions based on the output from a global climate model (GCM) (Wilks 1992, Barrow \& Semenov 1995, Wilks \& Wilby 1999, Hansen 2002, Dubrovsky et al. 2005). Daily scenarios are required by process-based models, which are used in the assessment of impacts of climate change on agricultural and ecological systems, as their input. Process-based models incorporate a mixture of non-linear interactions between their components and the environment (Porter \& Semenov 2005). Non-linearity in the model can lead to large differences in the model output in response to small variation in model inputs. For example, Porter \& Semenov (1999) demonstrated that daily scenarios derived from GCM that incorporated changes in climatic variability decreased mean wheat yield and significantly increased the risk of crop failure compared with scenarios that accounted only for changes in mean values. Non-linearity can also produce counterintuitive behaviours. In Semenov (2007), extreme weather events in the UK and extreme impacts on wheat were analysed for the set of daily scenarios based on predictions from the UK Climate Impacts Programme, known as UKCIP02 (Hulme et 
al. 2002). Analysis showed that, as the climate is getting warmer, extreme weather statistics, such as heat waves and summer drought, are likely to increase substantially in magnitude and frequency. Nevertheless, despite higher temperatures and lower summer precipitation for the future scenarios, the relative reduction in grain yield due to water stress was smaller than that for the present climate. This is because wheat matures earlier in a warmer climate, avoiding summer heat and severe drought stress.

Methodology for construction of daily site-specific scenarios for climate change studies is based on a stochastic weather generator and output from GCM (Wilks 1992, Semenov \& Barrow 1997, Wilks \& Wilby 1999). Calibrated with observed weather data at a site, the WG parameters are adjusted with predicted changes in climatic mean and variability, derived from the GCM output. This new parameter set is used by the WG to generate daily scenarios (Semenov 2007).

A WG should be rigorously tested before its application. A number of statistical tests are routinely used to assess the performance of WG at a site and compare similarities between synthetic and observed weather. A comparison of mean values and variances of climatic variables by applying the $t$-test and $F$-test is quite common. More detailed analysis of the distributions of climatic variables often employs Pearson's $\chi^{2}$-test or the Kolmogorov-Smirnov (K-S) test (Semenov et al. 1998, Wilks 1999, Qian et al. 2004). If synthetic weather is used as an input to a specific process-based model, e.g. a crop simulation or a hydrological model, then the performance of a WG could be tested by comparing the model outputs generated with observed and synthetic weather (Mavromatis \& Hansen 2001).

Frequency and magnitude of extreme weather events are likely to increase under climate change (Solomon et al. 2007). If WGs are going to be used to generate daily scenarios suitable for the analysis of extremes, then their ability to simulate weather extremes must be tested. However, only on a few occasions have such tests been carried out. Kysely \& Dubrovsky (2005) evaluated the Met\&Roll weather generator's ability to simulate extreme temperature events. They concluded that WGs based on the firstorder auto-regressive (AR) model have limited skill in reproducing most temperature-related extreme events for the climates of western and central Europe.

The aim of the present study was to assess the ability of the LARS-WG stochastic weather generator (available from www.rothamsted.bbsrc.ac.uk/mas-models/ lasrwg.php) to simulate extreme weather events at 20 locations with diverse climates. The following extreme events were selected for analysis: yearly maxima of daily precipitation, maximum temperature, and length of heat waves. LARS-WG is based on the series approach (Racsko et al. 1991), with a detailed description given in Semenov et al. (1998) and Semenov \& Brooks (1999). LARS-WG is site specific and produces synthetic daily time series of maximum and minimum temperature, precipitation and solar radiation. The weather generator distinguishes dry and wet days, depending on whether the precipitation is greater than zero. Precipitation is modelled using semi-empirical probability distributions for the lengths of wet and dry series and for the amount of precipitation on a wet day. A semi-empirical distribution is a histogram with a fixed number of intervals (10 in the case of LARS-WG). A semi-empirical distribution is sufficiently flexible and allows for the accurate simulation of various weather statistics (Semenov et al. 1998). Minimum temperature, maximum temperature and radiation are related to the amount of cloud cover, and so LARS-WG uses separate distributions for wet and dry days for each of these variables. The shape of the daily temperature distributions is approximated by the normal distribution, with the values of mean and standard deviation changing daily and calculated by a Fourier series. Time auto-correlations for minimum and maximum temperature are site specific, but constant throughout the year; the cross-correlation of the normalized residuals is pre-set at 0.6. Semi-empirical distributions for dry and wet days are used for solar radiation, because the observed distribution for radiation can deviate from the truncated normal distribution traditionally used in weather generators (Semenov et al. 1998, Hansen 1999, Parlange \& Katz 2000). LARS-WG has been used in various studies, including assessment of the impact of climate change (Barrow \& Semenov 1995, Barrow et al. 1996, Weiss et al. 2003, Lawless \& Semenov 2005, Khan et al. 2006, Scibek \& Allen 2006, Semenov 2007, Semenov \& Doblas-Reyes 2007).

In Section 2 the statistics of extreme events and the methodology for analysis are described. Section 3 presents a detailed analysis of extreme events at 20 locations and Section 4 a discussion of results. Section 5 presents the main conclusions.

\section{METHODS}

For our analysis we selected 20 sites with diverse climates, which represent a wide selection of ecoregion provinces (Bailey \& Hogg 1986, Bailey 1989), varying from continental tundra at Baker Lake, Canada, to desert at Boise, USA (Table 1). Geographically, the sites are located in Europe (10 sites), USA (5 sites), Canada (2 sites), Australia (1 site) and New Zealand (1 site). The number of years with observed weather varied between 30 and 56 yr (Table 1). Observed daily weather at each site was used by LARS-WG to com- 


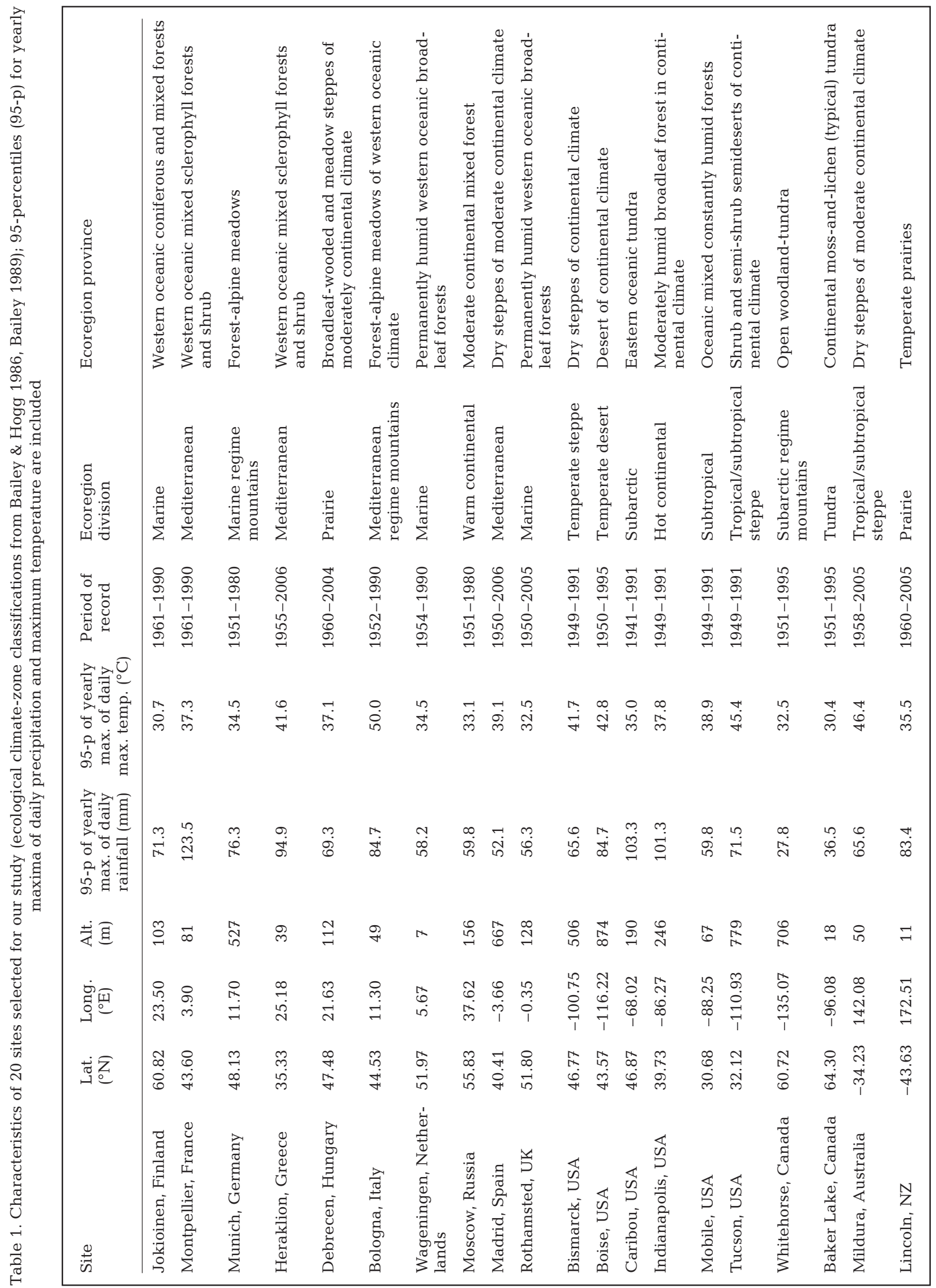


pute a site-specific set of parameters, which was used to generate $300 \mathrm{yr}$ of daily synthetic weather. Note that statistics of observed extreme weather events are not analysed by LARS-WG explicitly. To reproduce correctly the extreme weather events, the tails of distributions should be approximated well. If, for example, the observed temperature distribution deviates significantly from the normal distribution, then LARS-WG, which assumes normality of the distribution, is likely to fail to simulate extreme temperature accurately, although means and variances of daily temperature will be correctly reproduced.

The following extreme events were computed and compared for observed and synthetic daily weather: (1) yearly maxima of daily precipitation, (2) yearly maxima of daily maximum temperature and (3) yearly maxima of the length of heat waves. A heat wave is defined as a continuous period ( $2 \mathrm{~d}$ or more) with the daily maximum temperature $>30^{\circ} \mathrm{C}$. Isolated incidents of extreme high or low temperatures could seriously damage an agricultural plant. A continuous period of extremely high temperatures could be lethal, not only for crops, but also for humans (Basu et al. 2005). Summer 2003 was recorded as the hottest in Europe since 1500 (Luterbacher et al. 2004, Rebetez et al. 2006), with a heat-wave episode in August that led to an excess of 15000 deaths in France alone (Poumadere et al. 2005).

For each extreme event the following statistics were compared for observed and synthetic weather: (1) means of maxima and (2) return values for 10 and 20 yr periods. The return value of an extreme event for the $\mathrm{N}$-yr period is defined as the value $R V_{N}$, such that $R V_{N}$ is exceeded in any given year with a probability of $p=1 / N$, or, alternatively, the level that is expected to be exceeded on average once every $\mathrm{N}$ years (Coles 2001). The return level is derived from the generalized extreme value distribution (Eq. 1) by setting the cumulative distribution function equal to the desired probability $(1-p)$ and then solving for the return level. For observed data, the 95\% confidence intervals $\left(\mathrm{CI}^{95}\right)$ were calculated for means of maxima and return values. Confidence intervals were calculated by fitting the generalized extreme value distribution to the observed data and estimating standard errors of the parameters. If the extreme value calculated for synthetic data falls within the $\mathrm{CI}^{95}$ of the observed values, then we conclude that this statistic is simulated successfully at this site. We measured the skill of the weather generator by the number of sites where simulations of extreme weather events were successful.

To compute return values for 10 and $20 \mathrm{yr}$ periods we fitted the generalized extreme value (GEV) distribution to yearly maxima (Coles 2001, Beirlant et al. 2004). The GenStat 10 procedure was used to estimate 3 parameters $(\mu, \sigma, \xi)$ of the GEV distribution for observed and synthetic data (Payne et al. 2007):

$$
G(x ; \mu, \sigma, \xi)=\left\{\begin{array}{l}
\exp \left[-\left\{1+\xi \frac{(x-\mu)}{\sigma}\right\}_{+}^{-1 / \xi}\right], \xi \neq 0 \\
\exp \left[-\exp \left\{-\frac{(x-\mu)}{\sigma}\right\}\right], \xi=0
\end{array}\right.
$$

where $\sigma>0$ is the scale parameter and $\xi \in \Re$ is the shape parameter that governs the tail behaviour of the distribution. The sub-families defined by the value $\xi$ correspond, respectively, to the Gumbel $(\xi=0$, 'medium-tailed'), Fréchet $(\xi>0$ 'long-tailed') and Weibull $(\xi<0$, 'short-tailed') distributions.

The theory of extreme values is based on the Three Types Theorem, originally formulated without detailed proof by Fisher \& Tippett (1928) and later derived rigorously by Gnedenko (1943). Suppose we have a sequence of independent identically distributed (i.i.d.) random variables $\left\{Y_{i}, i=1, \ldots, n\right\}$. Let us denote the $n$-th sample maximum $M_{n}=\max _{1<i<n} Y_{i}$. The Three Types Theorem states that if a sequence of numbers $a_{n}$ and $b_{n}$ exists, such that $\frac{M_{n}-b_{n}}{a_{n}}$ converges to the nondegenerate distribution, then this distribution belongs to 1 of the 3 types: Gumbel, or Fréchet, or Weibull, which can be combined in the GEV distribution:

$$
P\left(\frac{M_{n}-b_{n}}{a_{n}} \leq x\right) \rightarrow G(x ; \mu, \sigma, \xi)
$$

The standardization with $a_{n}$ and $b_{n}$ is necessary since otherwise the $\operatorname{limit} \lim P\left(M_{n} \leq x\right)$ is trivial. The Three Types Theorem resembles the classical Central Limit Theorem, which states that the normalised sum of i.i.d. random variables converges to a standard normal distribution.

The estimation of parameters of the GEV distribution was performed by GenStat 10, using the maximumlikelihood estimation method (MLE). The standard asymptotic results of consistency, efficiency and normality hold if $\xi>-0.5$ (Smith 1985). Asymptotic consistency means that asymptotically there is no bias. Asymptotic efficiency means that asymptotically no unbiased estimator has a lower mean squared error. Asymptotic normality means that the MLE distribution converges to the normal distribution with the mean equal to the estimated parameters $(\mu, \sigma, \xi)$ and the covariance matrix equal to the inverse of the Fisher information matrix (Coles 2001). The square roots of the diagonal entries of this inverse matrix are estimates of the standard deviations of the 3 parameter estimates, known as the standard errors of those estimates. While these asymptotic properties only become strictly true in the limit of infinite sample size, in practice they are often assumed to be approximately true, especially when the sample size is large. In particular, inference about the estimated parameters is often based on the asymptotic normal distribution of the MLE. 


\section{RESULTS}

\subsection{Precipitation extremes}

Means of yearly maxima of daily precipitation were reproduced well by LARS-WG. For all sites, means computed for synthetic precipitation lay within the $\mathrm{CI}^{95}$ of means of the maxima for observed data (Fig. 1a). The largest difference between observed and generated means of precipitation maxima was $12 \mathrm{~mm}$ at Boise, USA. The discrepancies between observed and generated means of maxima at Boise could be explained by an abnormal shape of the precipitation distribution at Boise. Fig. 2 shows a cumulative probability distribution of daily precipitation for June based on records from 1940 to 1995 . Only $1 \%$ of daily precipitation exceeds $75 \mathrm{~mm}$, although the maximum daily precipitation for June from 1940 to 1995 was $191 \mathrm{~mm}$. LARS-WG approximates daily precipitation with a semi-empirical distribution consisting of a histogram with 10 intervals of increasing length (see Fig. 2, vertical dotted lines point to the intervals in a semi-
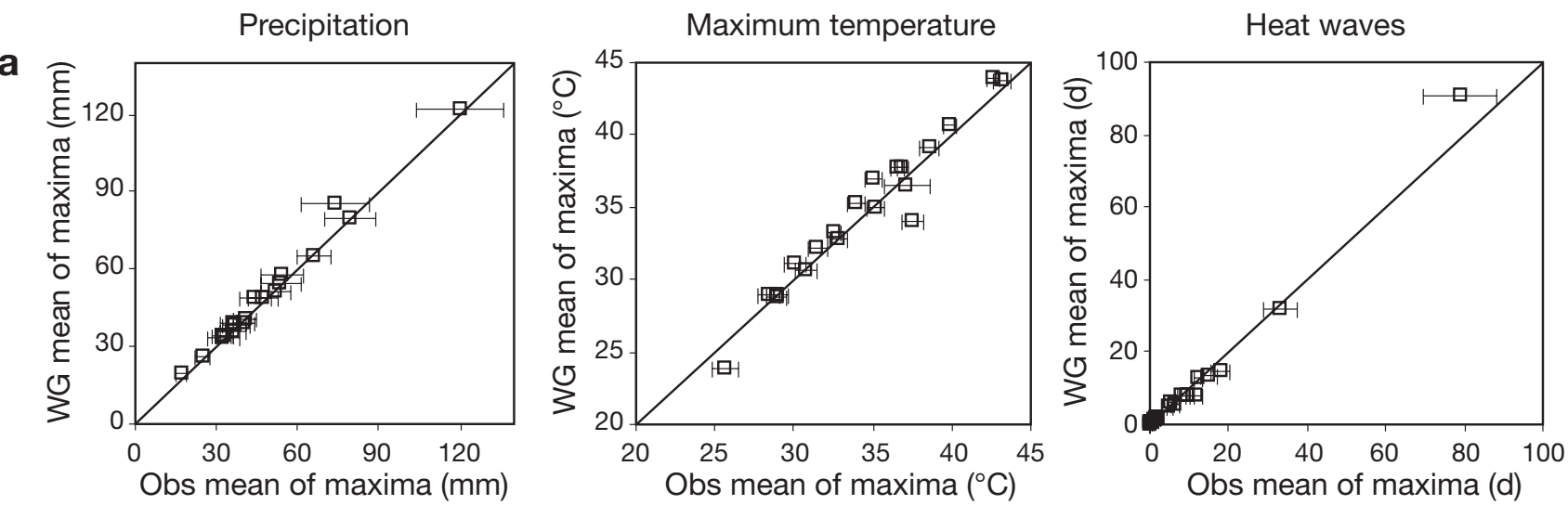

b
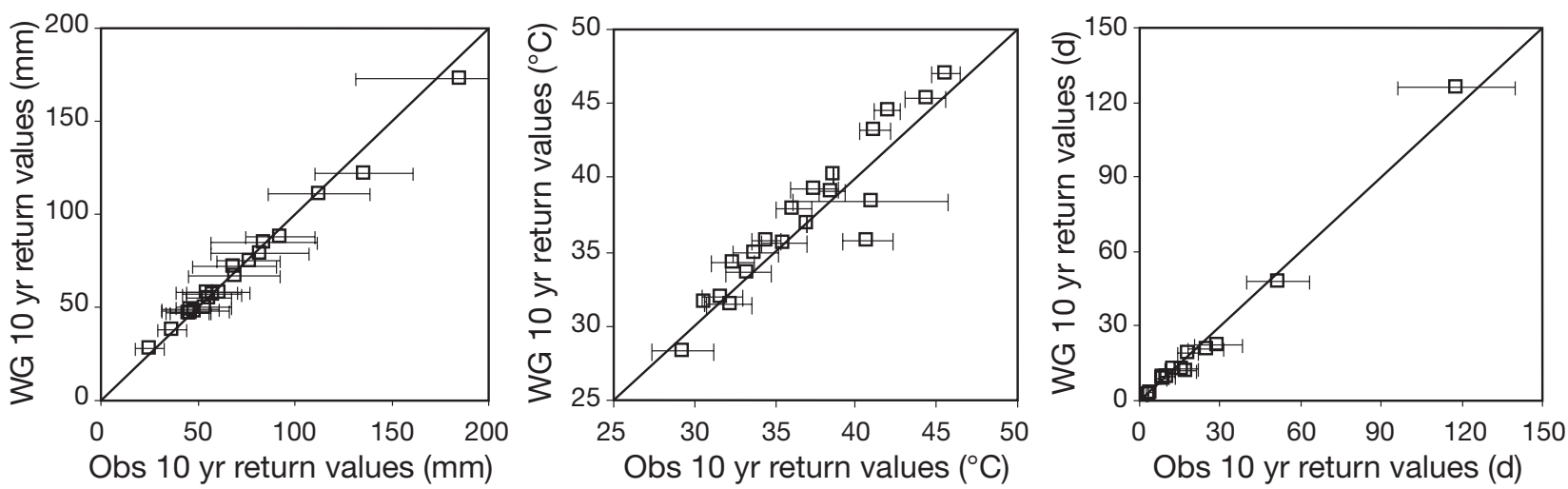

C
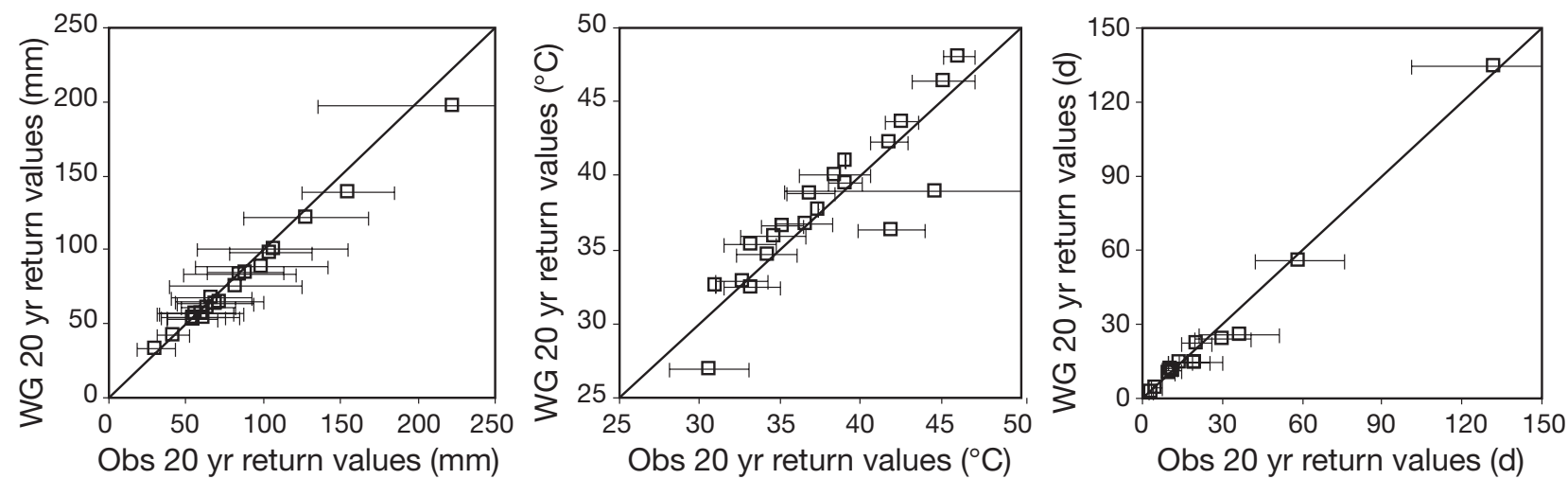

Fig. 1. Observed (obs) and synthetic (WG) extreme events for daily precipitation, daily maximum temperature and heat waves at 20 sites: (a) means of yearly maxima of daily values (b) $10 \mathrm{yr}$ and (c) $20 \mathrm{yr}$ return values. Bars are $95 \%$ confidence intervals for observed values 


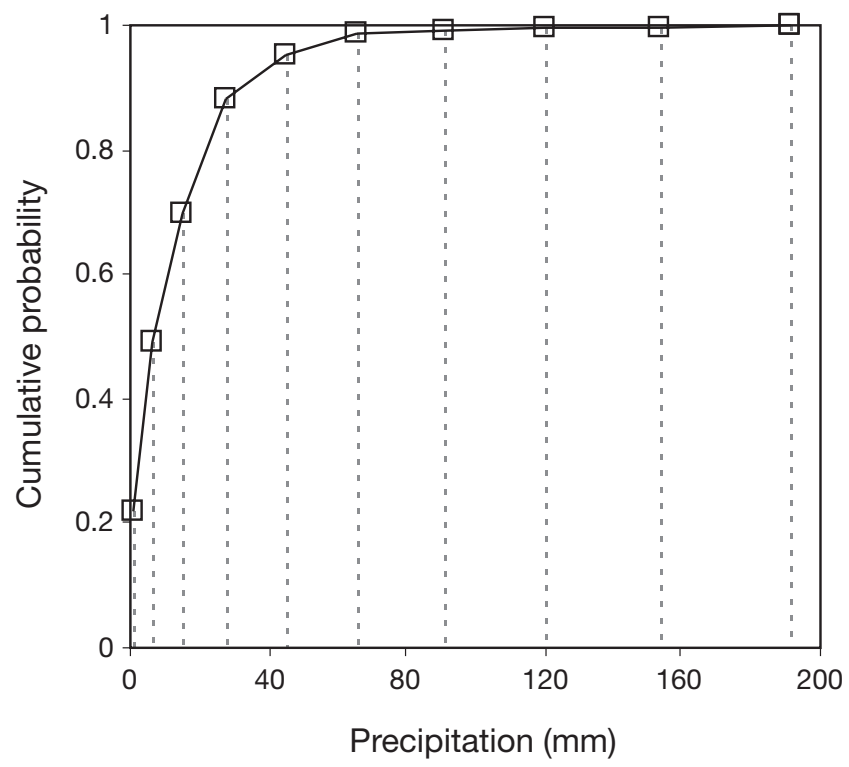

Fig. 2. Cumulative probability of daily precipitation as computed by LARS-WG for June at Boise, USA

empirical distribution). The range of observed precipitation at Boise is large, $191 \mathrm{~mm}$. As a result, intervals in semi-empirical distribution have to be large too, which reduces the accuracy of approximation, because within the intervals a cumulative probability function is approximated by a linear function (Fig. 2). In addition, 4 intervals of the semi-empirical distribution, covering the range between 80 and $200 \mathrm{~mm}$, account for $<1 \%$ of observations. The remaining $99 \%$ of observations have to be approximated by the 6 remaining intervals, resulting in further reduction in accuracy.

Return values for daily precipitation maxima for 10 and 20 yr periods were reproduced well by LARS-WG (Fig. 1b,c). All return values computed from synthetic data were within the $\mathrm{CI}^{95}$ of observed return values. The GEV distribution was fitted to the observed and synthetic data in order to estimate the return values. Due to the number of years with observed weather not being sufficiently large, 30 to $56 \mathrm{yr}$ depending on the site, uncertainties in calculating 10 and 20 yr return values were large. The $\mathrm{CI}^{95}$ for return values were, on average, $\pm 19 \mathrm{~mm}$ for the $10 \mathrm{yr}$ and $\pm 31 \mathrm{~mm}$ for the 20 yr return period. The largest differences between observed and generated return values were at Mobile, USA: $12 \mathrm{~mm}$ for the $10 \mathrm{yr}$ period and $25 \mathrm{~mm}$ for the $20 \mathrm{yr}$ period. The $10 \mathrm{yr}$ return value for precipitation at this site, calculated for observed data, was $185 \mathrm{~mm}$ with the $\mathrm{Cl}^{95}$ intervals $\pm 53 \mathrm{~mm}$; the return 20 yr value for precipitation was $225 \mathrm{~mm}$ with the $\mathrm{CI}^{95} \pm 87 \mathrm{~mm}$. The

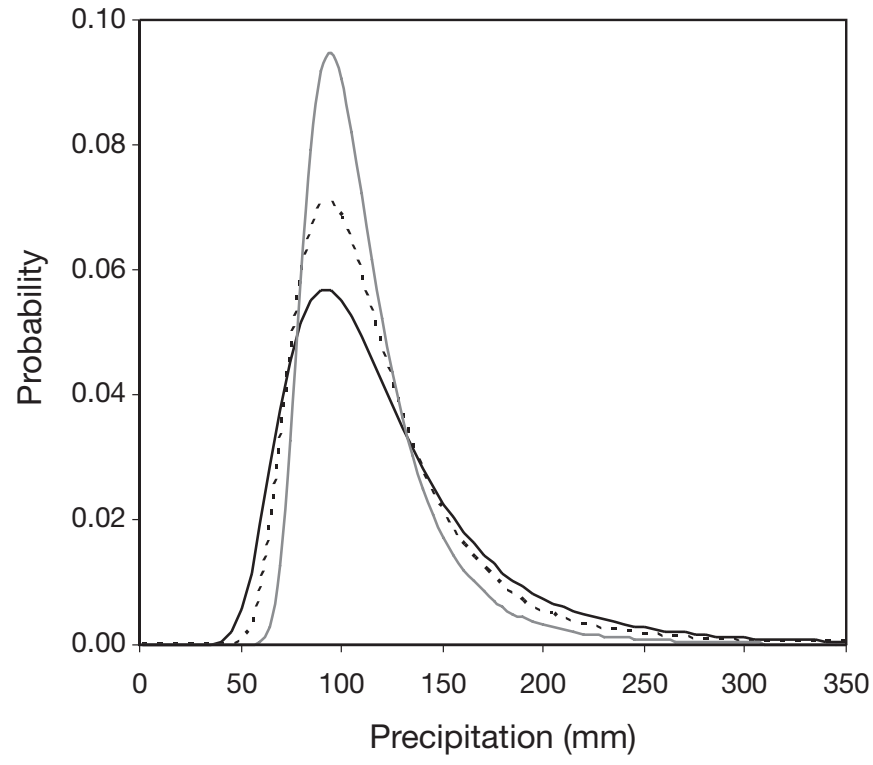

Fig. 3. Probability distribution functions for the generalized extreme value distribution fitted to observed yearly maxima of daily precipitation at Mobile, USA. Black solid line: parameter set $(\mu, \sigma, \xi)$, estimated using the maximum-likelihood estimation method; dashed line: parameter set $(\mu, \sigma-\mathrm{SE}, \xi)$; solid grey line: parameter set $(\mu, \sigma-2 \mathrm{SE}, \xi)$, where $\mathrm{SE}$ is a standard error. Values of parameters are in Table 2

large confidence intervals are the direct result of large standard errors for parameters estimated using the MLE method at Mobile (Table 2). For example, in Fig. 3 we present the GEV probability distributions for 3 sets of parameters: $(\mu, \sigma, \xi)$, an estimated set using the MLE method, and perturbed parameter sets $(\mu, \sigma-\mathrm{SE}, \xi)$ and $(\mu, \sigma-2 \mathrm{SE}, \xi)$, where SE is an estimated standard error. The tails of these distributions behave differently, resulting in the large differences in probabilities exceeding extreme thresholds, e.g. the probability to exceed $185 \mathrm{~mm}$ is 0.1 for the set $(\mu, \sigma-\mathrm{SE}, \xi)$ and 0.038 for the set $(\mu, \sigma-2 \mathrm{SE}, \xi)$.

\subsection{Maximum temperature extremes}

Means of yearly maxima for the maximum daily temperature were reproduced less accurately. Means of maxima calculated for synthetic temperature were out-

Table 2. Parameters of the generalized extreme value distribution with standard errors (in brackets) estimated by the maximum-likelihood estimation method for yearly maximum of daily precipitation at 3 sites

\begin{tabular}{|lccc|}
\hline Site & $\mu$ & $\sigma$ & $\xi$ \\
\hline Mobile, USA & $94.78(8.22)$ & $32.70(6.53)$ & $0.175(0.203)$ \\
Rothamsted, UK & $27.54(1.13)$ & $5.02(0.99)$ & $0.369(0.208)$ \\
Whitehorse, USA & $13.75(0.97)$ & $3.90(0.798)$ & $0.236(0.218)$ \\
\hline
\end{tabular}


side the $\mathrm{CI}^{95}$ of observed means for approximately half of the sites (Fig. 1a). However, root mean squared error (RMSE) between means of yearly maxima for observed and synthetic data was relatively small $\left(0.94^{\circ} \mathrm{C}\right)$, when Heraklion, where LARS-WG demonstrated poor performance, was excluded. The difference between means of maxima for observed and synthetic data at Heraklion was $3.6^{\circ} \mathrm{C}$. LARS-WG approximates normalised temperature residuals (after removing longterm mean and normalising variances) by the normal distribution. If the observed distribution differs from normal, then generated extreme values deviate from the observed, as at Heraklion (Fig. 4a). The GEV distribution fitted to the observed data will generate larger return values. Fig. 4b shows a quantile-quantile (Q-Q) plot for observed and synthetic maximum temperatures in July at Heraklion. The distribution for observed maximum temperature deviates substantially from the normal distribution, especially at both tails. A possible solution to this problem would be to use more flexible distribution, e.g. semi-empirical distribution, instead of the normal distribution to model maximum and minimum temperature.

The 10 and 20 yr return values are presented in Fig. 1b,c. Both 10 and 20 yr return values calculated for synthetic maximum temperature were within the $\mathrm{CI}^{95}$ of return values calculated for the observed data for approximately $70 \%$ of sites. The RMSE was relatively small for both 10 and 20 yr return values, 1.35 and $1.53^{\circ} \mathrm{C}$, respectively, if we exclude 2 sites, Heraklion and Bologna, where approximation of maximum temperature by the normal distribution produced poor results.

\subsection{Heat waves}

Means of yearly maxima for length of heat waves for synthetic weather were inside the $\mathrm{CI}^{95}$ for most of the sites. Only at 3 locations, i.e. Tucson, USA; Boise, USA; and Bologna, Italy, were the means of maxima calculated for synthetic data outside the $\mathrm{CI}^{95}$ for observed data (Fig. 1a).

Calculation of 10 and $20 \mathrm{yr}$ return values for heat waves was not possible for several sites where heat waves were very rare (or did not exist), e.g. Jokioinen, Finland, or Baker Lake, Canada. The MLE procedure did not converge for these sites. For the remaining sites, 10 and 20 yr return values for synthetic weather were within the $\mathrm{CI}^{95}$ of observed return values, with the exception of Bologna, Italy, for the $10 \mathrm{yr}$ return value (Fig. 1b, c). Analysis of maximum temperature at Bologna for July and August, the 2 hottest months, showed that the observed temperature distribution deviates considerably from the normal distribution at both tails of the distribution.

\section{DISCUSSION}

To model temperature, LARS-WG uses an assumption that the normalized residuals of maximum and minimum temperatures follow the standard normal distribution. The assumption is not universally true for the sites in this study. For those locations where maximum temperature did not follow the normal distribution, extreme value statistics derived from observed
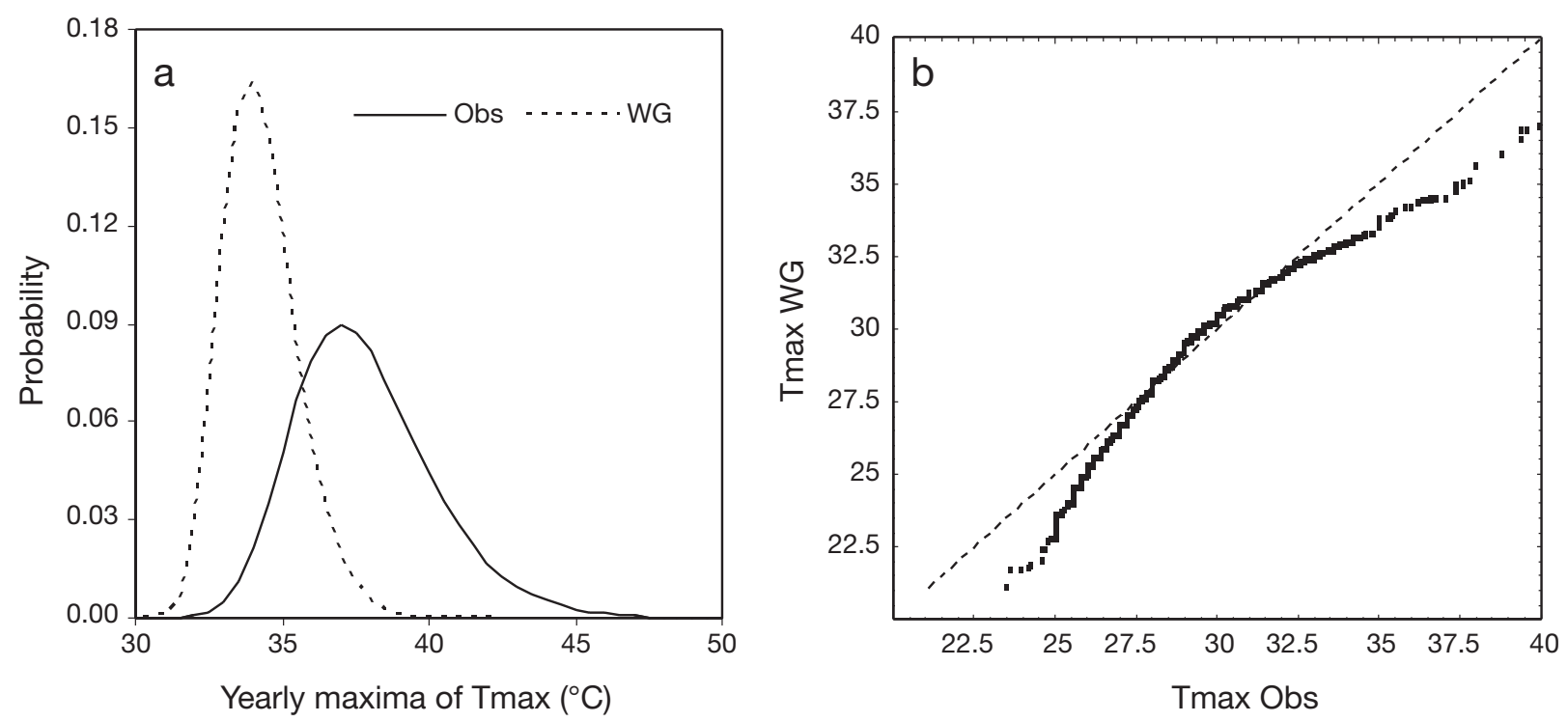

Fig. 4. (a) Generalized extreme value (GEV) distributions of yearly maxima of daily maximum temperature fitted to observed (solid line) and generated (dashed line) data at Heraklion, Greece; parameters $(\mu, \sigma, \xi)$ are $(36.56,2.06,-0.095)$ for observed and (33.49, $1.12,-0.120$ ) for generated data; (b) Q-Q plot of observed and generated maximum temperature in July at Heraklion, Greece 
and synthetic time series were significantly different. Non-normality of maximum and minimum temperature has been reported previously in Harmel et al. (2002), where authors analysed the adequacy of generating temperature data from the normal distribution. The assumption of normality in observed daily temperatures (used by many weather generators, including WGEN: Richardson \& Wright 1984; CLIGEN: Johnson et al. 1996; Met\&Roll: Kysely \& Dubrovsky 2005), was evaluated by testing the hypothesis that daily minimum and maximum temperatures are normally distributed for each month. Based on these analyses, it was concluded that daily maximum and minimum temperatures are generally not normally distributed in each month, but are often slightly skewed.

One of the possible solutions to improve the simulation of temperature would be to replace the normal distribution with a more flexible distribution, e.g. a semi-empirical distribution, in a similar way to that used in LARS-WG for precipitation, where Gamma or mixed-exponential distributions were replaced with a semi-empirical distribution. As was reported by Qian et al. (2004), using empirical distributions to approximate normalized temperature residuals in the AAFCWG weather generator helped to improve simulation of maximum and minimum temperatures in cases where observed temperatures did not follow the normal distribution.

A semi-empirical distribution is flexible. The drawback of this flexibility is that in some cases a semi-empirical distribution can 'overfit' the observed data. Overfitting is a typical problem encountered when a statistical distribution or model has many parameters and high complexity in comparison to the amount of data available. In cases where a semi-empirical distribution is fitted to a dataset with an outlier, its parameters may overestimate the significance of this single event, because the number of intervals in the semiempirical histogram is fixed. At Mobile, USA, the highest daily precipitation of $339 \mathrm{~mm}$ was recorded in April 1955 (second highest precipitation was $209 \mathrm{~mm}$ ). When semi-empirical distributions were fitted to observed daily precipitation in April at Mobile with and without this unique precipitation event, probability density functions showed noticeable differences for precipitation in the range from 0 to $15 \mathrm{~mm}$. It is likely that the outlier, $339 \mathrm{~mm}$, comes from a different distribution than the rest of the sample and, therefore, should be modelled separately.

WGs tend to underestimate inter-annual variability for climatic variables (Katz \& Parlange 1998, Wilks \& Wilby 1999, Hansen \& Mavromatis 2001). It was reported in Semenov et al. (1998) that LARS-WG tends to underestimate the variance in monthly means of some weather variables. This is because the model has only a simple auto-correlation structure, and the observed data contain many periods in which successive values of precipitation or temperature are highly correlated, whereas the synthetic data vary more randomly. Underestimation of variance for monthly means does not necessarily affect simulation of extremes, because the extreme events studied here were formulated in terms of daily values in comparison with cumulative values for monthly means. In Fig. 5 maxima and minima of monthly means of maximum temperature for observed and synthetic weather are presented for each month of the year along with the monthly standard deviations at Bismarck, USA. The F-test for monthly variances showed that 8 out of 12 monthly variances calculated for synthetic data significantly underestimated the observed values. Nevertheless, extreme statistics and return values for maximum temperature and heat waves were reproduced accurately at Bismarck.

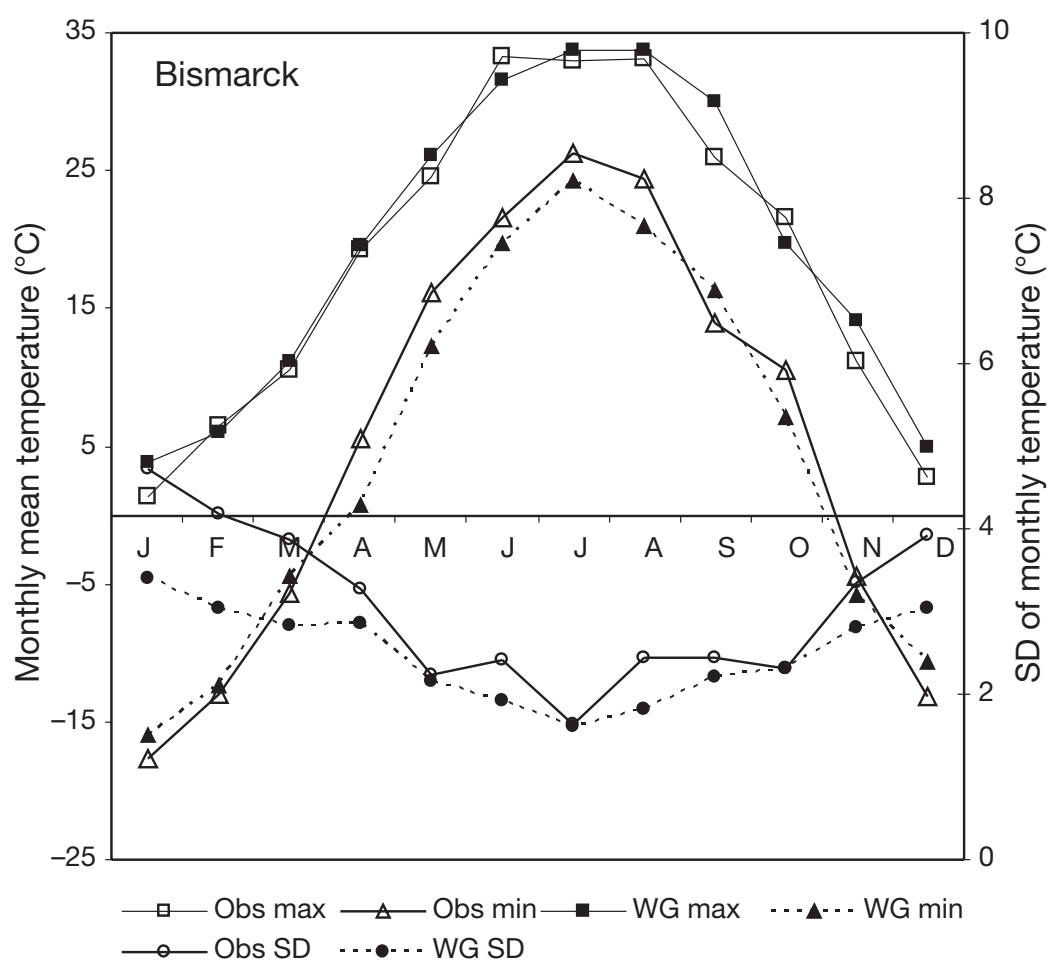

Fig. 5. Maxima (max) and minima (min) of monthly means and monthly variances (SD) of maximum temperature for observed (obs) and synthetic (WG) weather at Bismarck, USA 


\section{CONCLUSIONS}

The skill of LARS-WG to simulate extreme weather events was tested at 20 sites with diverse climates. Extreme events include yearly maxima of daily precipitation, maximum temperature and length of heat waves. The means of yearly maxima for daily precipitation and 10 and 20 yr return values for precipitation were reproduced accurately for all sites. Means of yearly maxima for daily maximum temperature were reproduced less accurately; for approximately half of the sites the means of the maxima calculated for synthetic maximum temperature were outside the $\mathrm{CI}^{95}$ of observed means. However, RMSE between means of temperature maxima for observed and synthetic data was relatively small, $0.94^{\circ} \mathrm{C}$, if 1 site, Heraklion, Greece, where LARS-WG demonstrated poor performance, was excluded. The means of yearly maxima for the lengths of heat waves and 10 and 20 yr return values were reproduced accurately for sites where heat waves are common. A possible solution to improve the simulation of extreme temperature events would be to use a more flexible distribution for temperature, e.g. a semi-empirical distribution.

Acknowledgements. Many thanks to Adrian Price and Sue Welham for their comments. I acknowledge the contribution of anonymous reviewers. Research was supported by the Department of Environment, Food and Rural Affairs of the United Kingdom (Project AC0301). Rothamsted Research receives grant-aided support from the Biotechnology and Biological Sciences Research Council of the United Kingdom.

\section{LITERATURE CITED}

Bailey RG (1989) Explanatory supplement to ecoregions map of the continents. Environ Conserv 16:307-309

Bailey RG, Hogg HC (1986) A world ecoregions map for resource reporting. Environ Conserv 13:195-202

Barrow EM, Semenov MA (1995) Climate change scenarios with high spatial and temporal resolution for agricultural applications. Forestry 68:349-360

Barrow E, Hulme M, Semenov MA (1996) Effect of using different methods in the construction of climate change scenarios: examples from Europe. Clim Res 7:195-211

Basu R, Dominici F, Samet JM (2005) Temperature and mortality among the elderly in the United States: a comparison of epidemiologic methods. Epidemiology 16:58-66

Beirlant J, Geogebeur Y, Segers J, Teugels J (2004) Statistics of extremes. Theory and applications. John Wiley \& Sons, Chichester

Coles S (2001) An introduction to statistical modelling of extreme values. Springer-Verlag, London

Dubrovsky M, Nemesova I, Kalvova J (2005) Uncertainties in climate change scenarios for the Czech Republic. Clim Res 29:139-156

Fisher RA, Tippett LHC (1928) Limiting forms of the frequency distributions of the largest or smallest member of a sample. Proc Camb Philol Soc 24:180-190

Gnedenko BV (1943) Sur la distribution limite du terme maximum d'une serie aleatoire. Ann Math 44:423-453
Hansen JW (1999) Stochastic daily solar irradiance for biological modelling applications. Agric For Meteorol 94:53-63

Hansen JW (2002) Realizing the potential benefits of climate prediction to agriculture: issues, approaches, challenges. Agric Syst 74:309-330

Hansen JW, Mavromatis T (2001) Correcting low frequency variability bias in stochastic weather generators. Agric For Meteorol 109:297-310

Harmel RD, Richardson CW, Hanson CL, Johnson GL (2002) Evaluating the adequacy of simulating maximum and minimum daily air temperature with the normal distribution. J Appl Meteorol 41:744-753

Hulme M, Jenkins GJ, Lu X, Turnpenny JR and others (2002) Climate change scenarios for the United Kingdom: the UKCIP02 scientific report. Tyndall Centre for Climate Change Research and School of Environmental Sciences, University of East Anglia, Norwich

Johnson GL, Hanson CL, Hardegree SP, Ballard EB (1996) Stochastic weather simulation: overview and analysis of two commonly used models. J Appl Meteorol 35: 1878-1896

Katz RW, Parlange MB (1998) Overdispersion phenomenon in stochastic modelling of precipitation. J Clim 11:591-601

Khan MS, Coulibaly P, Dibike Y (2006) Uncertainty analysis of statistical downscaling methods using Canadian Global Climate Model predictors. Hydrol Process 20:3085-3104

Kysely J, Dubrovsky M (2005) Simulation of extreme temperature events by a stochastic weather generator: effects of interdiurnal and interannual variability reproduction. Int J Climatol 25:251-269

Lawless C, Semenov MA (2005) Assessing lead-time for predicting wheat growth using a crop simulation model. Agric For Meteorol 135:302-313

Luterbacher J, Dietrich D, Xoplaki E, Grosjean M, Wanner H (2004) European seasonal and annual temperature variability, trends and extremes since 1500. Science 303: 1499-1503

Mavromatis T, Hansen JW (2001) Interannual variability characteristics and simulated crop response of four stochastic weather generators. Agric For Meteorol 109: 283-296

Parlange MB, Katz RW (2000) An extended version of the Richardson model for simulating daily weather variables. J Appl Meteorol 39:610-622

Payne RW, Harding SA, Murray DA, Soutar DM and others (2007) The guide to GenStat Release 10, Part 2: Statistics. VSN International, Hemel Hempstead

- Porter JR, Semenov MA (1999) Climate variability and crop yields in Europe. Nature 400:724

Porter JR, Semenov MA (2005) Crop responses to climatic variability. Philos Trans R Soc B 360:2021-2035

> Poumadere M, Mays C, Le Mer S, Blong R (2005) The 2003 heat wave in France: dangerous climate change here and now. Risk Anal 25:1483-1494

Qian BD, Gameda S, Hayhoe H, De Jong R, Bootsma A (2004) Comparison of LARS-WG and AAFC-WG stochastic weather generators for diverse Canadian climates. Clim Res 26:175-191

$>$ Racsko P, Szeidl L, Semenov M (1991) A serial approach to local stochastic weather models. Ecol Modell 57:27-41

> Rebetez M, Meyer H, Dupont O, Schindler D, Gartner K, Kropp JP, Menzel A (2006) Heat and drought 2003 in Europe: a climate synthesis. Ann For Sci 63:569-577

Richardson CW, Wright DA (1984) WGEN: a model for generating daily weather variables. Rep ARS-8, U.S. Department of Agriculture, Agricultural Research Service, Washington, DC 
Scibek J, Allen DM (2006) Modelled impacts of predicted climate change on recharge and groundwater levels. Water Resour Res 42:W11405 (available at: www.agu.org/pubs/ crossref/2006/2005WR004742.shtml)

Semenov MA (2007) Development of high resolution UKCIP02-based climate change scenarios in the UK. Agric For Meteorol 144:127-138

Semenov MA, Barrow EM (1997) Use of a stochastic weather generator in the development of climate change scenarios. Clim Change 35:397-414

Semenov MA, Brooks RJ (1999) Spatial interpolation of the LARS-WG stochastic weather generator in Great Britain. Clim Res 11:137-148

Semenov MA, Doblas-Reyes FJ (2007) Utility of dynamical seasonal forecasts in predicting crop yield. Clim Res 34:71-81

Semenov MA, Brooks RJ, Barrow EM, Richardson CW (1998) Comparison of the WGEN and LARS-WG stochastic weather generators for diverse climates. Clim Res 10: 95-107

Editorial responsibility: Eric DeWeaver, Madison, Wisconsin, USA
Smith RL (1985) Maximum likelihood estimation in a class of nonregular cases. Biometrika 72:67-90

Solomon S, Qin D, Manning M, Marquis M and others (eds) (2007) Climate change 2007: the physical science basis contribution of Working Group I to the fourth assessment report of the Intergovermental Panel on Climate Change. Cambridge University Press, New York

Weiss A, Hays CJ, Won J (2003) Assessing winter wheat responses to climate change scenarios: a simulation study in the U.S. Great Plains. Clim Change 58:119-147

Wilks DS (1992) Adapting stochastic weather generation algorithms for climate changes studies. Clim Change 22: 67-84

Wilks DS (1999) Multisite downscaling of daily precipitation with a stochastic weather generator. Clim Res 11:125-136

Wilks DS, Wilby RL (1999) The weather generation game: a review of stochastic weather models. Prog Phys Geogr 23: 329-357

Submitted: April 28, 2007; Accepted: January 2, 2008

Proofs received from author(s): February 1, 2008 